\title{
The Dynamics of Abnormal Grain Growth in a Particle Containing System: New Insights from Multimodal Three-Dimensional X-Ray Imaging
}

\author{
Jiwoong Kang ${ }^{1 *}$, Ning Lu ${ }^{1}$, Nancy Senabulya ${ }^{2}$, Nicolas Gueninchault ${ }^{3}$ and Ashwin J. Shahani ${ }^{*}$ \\ 1. Department of Materials Science and Engineering, University of Michigan, Ann Arbor, MI, USA. \\ 2. Michigan Center for Materials Characterization, University of Michigan, Ann Arbor, MI, USA. \\ 3. Xnovo Technology ApS, Køge, Denmark. \\ *Corresponding authors: kangjw@umich.edu, shahani@umich.edu
}

The vast majority of engineering materials are in form of polycrystals, which are composed of grains (crystallites) with different sizes, shapes, and orientations. Since the intrinsic grain structure of a material has significant impact on its properties, many investigators have been devoted to studying the dynamics of the grain network under various external stimuli, e.g., heat. In this scenario, grain growth is initiated during an isothermal anneal in two different ways. In the first, normal grain growth occurs when grains grow continuously, reaching a unimodal grain size distribution in the steady-state. A second route of grain coarsening is through abnormal grain growth (AGG), a discontinuous phenomenon in which few grains grow more rapidly compared to other grains, resulting in a bimodal distribution of grain size. In a few cases, the abnormal grain consumes the entire microstructure, resulting in a single crystal [1]. AGG occurs in many polycrystalline materials, yet its origin remains unclear [2, 3].

Past experimental studies on capturing AGG have relied heavily on two-dimensional (2D) experiments, such as surface characterization via electron backscatter diffraction (EBSD) and optical microscopy. However, such experiments have had limited success in developing a comprehensive model of AGG due to lack of information below the sample surface. Furthermore, these characterization techniques are destructive in nature, and thus it is impossible to follow the individual grain trajectories as a function of time (in the same specimen). Integrating X-ray absorption contrast tomography (ACT) with X-ray diffraction contrast tomography (DCT) in the laboratory can provide in-depth details on microstructures that are simultaneously polycrystalline and multiphase. For instance, recently, Keinan et al. (2018) analyzed the heterogeneous microstructure of metallurgical-grade polycrystalline silicon — including the grain boundaries, impurity particles, and their correlations - through this approach, providing a unified three-dimensional (3D) description of the sample [4]. Here, we have adopted this novel method to gain new insights in situ into the problem of AGG in a particle-containing system.

An alloy of composition $\mathrm{Al}-3.5 \mathrm{wt} \% \mathrm{Cu}$ was used as a model system for investigating AGG. At a temperature of $485^{\circ} \mathrm{C}$, the sample exists in the form of $\theta-\mathrm{Al}_{2} \mathrm{Cu}$ particles in a polycrystalline $\mathrm{Al}$ matrix. The ingot was cold rolled to a $50 \%$ thickness reduction before it was machined via EDM into a cylindrical specimen suitable for X-ray tomographic imaging in the laboratory. To ensure that the sample was fully recrystallized, it was annealed at $400^{\circ} \mathrm{C}$ for $30 \mathrm{~min}$ prior to imaging. The prepared specimen was then imaged through DCT on a laboratory X- ray microscope (Zeiss Xradia 520 Versa) at the Michigan Center for Materials Characterization. Due to the non-destructive nature of this technique, we were able to image the microstructural evolution in an interrupted manner, whereby DCT data was collected when the isothermal anneal (at $485^{\circ} \mathrm{C}$ ) was paused. Ultimately, DCT scans were done at five time intervals $(0,22,42,67$, and $97 \mathrm{~min}$.). The specimen was annealed an additional $90 \mathrm{~min}$. and imaged in ACT to identify the distribution of secondary phase $\theta$ particles within the tomographic field-of-view. 
Fig. 1 shows 3D grain maps of the specimen as a function of time. An abnormal grain (in blue) was observed after 42 minutes of annealing; this grain engulfed the vast majority of the sample volume at the final annealing state $(97 \mathrm{~min}$.). The 3D data provided an unprecedented opportunity to uncover what determines the expansion and shrinkage of grains — including the abnormal grain — as a function of the initial grain size and topology [5,6]. However, both of these metrics do not correlate with grain growth rate. Instead, we find a strong correlation between the locations of secondary phase particles (obtained via ACT) and grain boundaries (imaged via DCT). Fig. 2 (a)-(d) shows our quantification of the relationship between particle and grain boundary distributions. We find that as the specimen is annealed, the distribution of grain boundaries becomes more closely correlated to the distribution of $\theta$ particles, suggesting that the abnormal grain nucleates in a relatively particle-free region of the sample volume. Meanwhile, the normal grains are pinned by a dense particle cloud. Further work is underway to determine why the abnormal grain persists to consume the sample volume.

\section{References:}

[1] T Kusama et al., Nat. Commun. 8 (2017), p. 354.

[2] T Omori, H Iwaizako and R Kainuma, Mater. Des. 101 (2016), p. 263.

[3] H Park et al., Mater. Charact. 146 (2018), p. 204.

[4] R Keinan et al., Acta Mater. 148 (2018), p. 225.

[5] M Hillert, Acta Metall. 13 (1965), p. 227.

[6] WW Mullins, Acta Metall. 37 (1989), p. 2979.

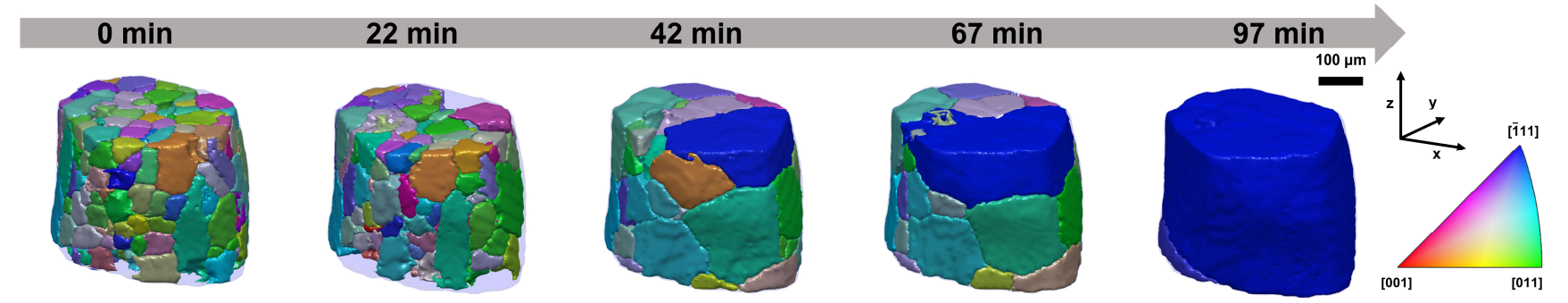

Figure 1. 3D grain maps of $\mathrm{Al}-3.5 \mathrm{wt} \% \mathrm{Cu}$ sample along the specified annealing states at $485{ }^{\circ} \mathrm{C}$. Grain color corresponds to crystallographic direction parallel to the $\mathrm{z}$ direction


Figure 2. Quantification of the relationship between particle and grain boundary distributions. (a) Locations of grain boundaries and $\theta$ particles at $0,22,42$, and $67 \mathrm{~min}$, averaged over the sample height (see schematic at left). Frequency represents the number of $\theta$ particles and grain boundary voxels per sector volume, respectively. (b) Pearson correlation coefficient of the two during the anneal. 